M. Pierrot, M. Blaise, S. Hugon, F. Bonnel, M. Cupa

\title{
Interférences hémo- dynamiques entre le diltiazem et le thiopental - étude expérimentale chez le porc
}

La prescripion d' agents bloqueurs calciques connair un développement croissant. Les barbituriques interferent sur les mouvements calcigues cellulaires. Ce travail ésudie l'intéraction hémodynamique, chez le porc, entre le Thiopental et le Dilitazem. injecté à la dose de $0,15 \mathrm{mg} \cdot \mathrm{kg}^{-1}$ puis perfusé à débit constant a 0,07 $m g \cdot k g^{-1} \cdot h^{-1}$.

De la cinquieme à la dixième minute, la diminution de la post charge s'associe à une déprèssion modérée de la contractilité du ventricule gauche. Ces variables retrouvent leurs valeurs initiales en 30 minutes. La fréquence et le débit cardiaque ne varient pas, Leur stabilitê serail le résultat d'interférences complexes entre l'effet de chacune de deux drogues sur le systeme sympathique et la mécanique ventriculaire.

\section{Key words}

HEART, ARRHYTHMIAS: calcium channel blockers, diltiazem; ANAESTHETICS INTRAVENous: thiopental.

Le Diltiazem* fait partie de la classe hétérogène des agents bloqueurs calciques qui inhibent les transferts membranaires du calcium, dépendant du potentiel d'action., ${ }^{1,2}$ Ils provoquent ainsi la relaxation, des muscles lisses vasculaires et myocardiques striés et un effet inotrope négatif d'intensité variable suivant l'agent considéré. Les bloqueurs calciques trouvent, ainsi leur indication première dans la

*Tildiemr: Laboratoire DAUSSE - L.E.R.S. (Paris).

Laboratoirc d'Anesthésiologie Expérimentale, U.E.R. Biomédicale de Bobigny, Université Paris XIII, 74, Rue Marcel Cachin - 93000 Bobigny. maladie coronarienne car ils réduisent la consommation d'oxygène du myocarde en diminuant, tout au moins, les conditions de charge et de travail du ventricule gauche.

Sachant que les effets, dose dépendants, inotrope négatif et vasodilatateurs des barbituriques sont aussi rapportés à une altération des mouvements cellulaires du calcium, ${ }^{3,4}$ nous nous sommes proposés ici de rechercher une éventuelle interférence hémodynamique entre le Diltiazem et une anesthésie réalisée par le Thiopental.

\section{Materiel et méthodes}

Sept porcs domestiques (1/2 Large White, 1/2 Landrace Français) ont été utilisés pour cette étude; 5 femelles et 2 mâles castrés, âgés de 3 mois et pesant $23 \mathrm{~kg} \pm 2,3$. Sur un animal à jeun depuis 24 heures, l'anesthésie est induite par le Thiopental à un pour cent par voie intra-museulaire (100 $\mathrm{mg} \cdot \mathrm{kg}^{-1}$ ) puis entretenue par perfusion à débit constant ( $\left.50 \mathrm{mg} \cdot \mathrm{kg}^{-1} \cdot \mathrm{h}\right)$, de la veine marginale de l'oreille. Au stade chirurgical de l'anesthésie caractérisée par la perte du clignement des paupières, l'électrocardiogramme étant surveillé cn $\mathrm{D} 2$, une trachéotomie est réalisée et la ventilation assurée avec un respirateur volumétrique (RPR) $\mathrm{FiO}_{2}=$ 0,$4 ; F R=12 \pm 3 ; \forall t=550 \pm 45 \mathrm{ml}$.

La préparation hémodynamique dure en moyenne 50 minutes. Elle expose chirurgicalement les vaisseaux du cou et pratique un cathétérisme des cavités droites et gauches du cour. Le cathétérisme droit, via la veine jugulaire droite, positionne une sonde de $S$ wan-Ganz à thermistance distale (5 F) dans l'artère pulmonaire. Au moyen de deux capteurs de pression Sthatam P23 dB, elle permet la 
TABLEAU I Résultats hemodynamiques de l'association thiopental-Diltiazem

\begin{tabular}{|c|c|c|c|c|c|c|}
\hline & & Base To & $5^{\prime}$ & $10^{\prime}$ & $20^{\prime}$ & $30^{\prime}$ \\
\hline Fréquence cardiaque & batt/min & $118,8 \pm 6,6$ & $111,4 \pm 5,5$ & $110,7 \pm 11,3$ & $111,7 \pm 6,2$ & $112,4 \pm 7,4$ \\
\hline Débit cardiaque & $1 / \min$ & $3,76=0,78$ & $3,48 \pm 0,83$ & $3,77 \pm 0,72$ & $3,75 \pm 0,64$ & $3,88 \pm 0,87$ \\
\hline VES & $1 / \min$ & $31 \pm 5,3$ & $31,2 \pm 7,3$ & $34 \pm 6,9$ & $33,6 \pm 6,1$ & $34,6 \pm 8,3$ \\
\hline PAOM & $\mathrm{mmHg}$ & $120,5 \pm 6,6$ & $84 \pm 18,8 f$ & $106,4=12,1^{*}$ & $112,8=5,27^{*}$ & $115 \pm 5,4$ \\
\hline PAOS & $\mathrm{mmH}$ & $142,3 \pm 8,5$ & $106,8 \pm 20,5 \dagger$ & $126 \pm 8,76 \dagger$ & $133,3 \pm 8^{*}$ & $136,8 \pm 5,5$ \\
\hline PAOD & $\mathrm{mmHg}$ & $111,3 \pm 6,5$ & $72,4 \pm 20,1 \dagger$ & $96,5 \pm 18,6^{*}$ & $101,4 \pm 4,4 \dagger$ & $105,3 \pm 4,8$ \\
\hline PTDVG & $\mathrm{mmHg}$ & $5,8 \pm 0,2$ & $5,4 \pm 0,3$ & $5,1 \pm 0,3$ & $4,8 \pm 0,4$ & $4,7 \pm 0,3$ \\
\hline RAS & Dynes's $\mathrm{s}^{-1} \cdot \mathrm{cm}^{-5}$ & $2565=422$ & $1857 \pm 333 \dagger$ & $2225 \pm 412$ & $2380=378$ & $2374 \pm 444$ \\
\hline $\mathrm{dP} / \mathrm{dt} \max$ & $\mathrm{mmHg} \cdot \mathrm{sec}^{-1}$ & $1643 \pm 117$ & $1157 \pm 268 \ddagger$ & $1371 \pm 2277$ & $1528=111$ & $1557=100$ \\
\hline WSVG & $\mathrm{gm}$ & $47,8 \pm 8,6$ & $34 \pm 12,7 \ddagger$ & $46,3 \pm 9$ & $49,3 \pm 8$ & $51,8 \pm 13$ \\
\hline
\end{tabular}

VES: volume d'éjection systolique; PAOM: pression aortique moyenne; PAOS: pression aorique systolique; PAOD: pression aortique diastolique; PTDVG: pression télédiastolique du ventricule gaựe; RAS: résistances artérielles systémiques totales; dP/dt dérive première de la pression ventriculaire en phase iso-volumétrique; WSVG: Iravail systolique du ventricule gauche. Significativité du T-Test par rapport à la base: ${ }^{*} \mathrm{p}<0,05 ; \ddagger \mathrm{p}<0,01 ; \ddagger \mathrm{p}<0,001$.

mesure des pressions artérielles pulmonaires et auriculaires droites; le débit cardiaque est mesuré par la méthode de thermodilution au moyen d'un calculateur Edwards Lab. $9520 \mathrm{~A}$.

Le cathétérisme gauche utilise deux sondes à micromanomètre distal ICEL, la sonde $7 \mathrm{~F}$ à lumen latéral est positionnée dans le ventricule gauche (VG) via la carotide gauche: la sonde $5 \mathrm{~F}$ mesure les pressions de l'aorte thoracique via la carotide droite. Les signaux de pression sont amplifiés par des modules CGR Telco (M52) et celui de la sonde ICEL 7 est intégré pour le calcul de la dP/dt (DE 53); ils sont visualisés sur un oscilloscope (05-283) et enregistrés sur papier UV sensible à l'aide d'un appareil Bell et Howell 6 pistes (Def 035-154).

\section{Protocole d'étude}

L'étude est conduite à thorax fermé. Une fois la préparation hémodynamique réalisée, l'animal est laissé au repos 45 minutes, au terme desquelles les gaz du sang artériels sont vérifiés et les mesures hémodynamiques sous Thiopental enregistrées (To). Le Diltiazem est injceté en bolus $\left(0,15 \mathrm{mg} \cdot \mathrm{kg}^{-1}\right)$ puis perfusé à débit constant $\left(0,07 \mathrm{mg} \cdot \mathrm{kg}^{-1} \cdot \mathrm{h}\right)$ par la sonde ICEL $7 \mathrm{~F}$.

A $5,10,20,30$ minutes, les variables suivantes sont enregistrées: fréquence cardiaque ( $\mathrm{Fc}=$ batt $/$ $\mathrm{min})$; débit cardiaque $(\mathrm{Qc}=1 / \mathrm{min})$; les pressions aortiques systolique (PAOS $=\mathrm{mmHG}$ ), diastolique $(\mathrm{PAOD}=\mathrm{mmHG})$, moyenne $(\mathrm{PAOM}=\mathrm{mmHG})$ et télédiastolique du ventricule gauche (PDTVG = $\mathrm{mmHG}$ ) ainsi que la dérivée première en fonction du temps de la pression ventriculaire gauche en phase iso-volumétrique ( $\mathrm{dP} / \mathrm{dt}=\mathrm{mmHg} / \mathrm{sec}^{-1}$ ); sont alors calculés les volumes d'éjection systolique (VES $=\mathrm{Qc} / \mathrm{Fc}: \mathrm{ml} / \mathrm{batt}$ ), le travail systolique du ventricule gauche (WSVG $(\mathrm{gm})=(P A O M-$ PTDVG) $\times($ VES $) \times 1,36 / 1000)$ et les résistances artérielles systémiques (PAOM - POD) Qc $\times 80=$ dynes $/ \mathrm{s}^{-1} / \mathrm{cm}^{-5}$ ).

L'analyse statistique des résultats utilise le TTest apparié de Student sur la différence des moyennes; chaque animal étant son propre témoin. La significativité des variations extraite des tables de Fisher et Yates est retenue pour $\mathrm{P}<0,05$.

\section{Résultats}

La température centrale ne varie pas de façon significative au cours du protocole. La Vérification des gaz du sang artériel est satisfaisante: $\mathrm{PO}_{2}=$ $16,7 \pm 1,38 \mathrm{KP}, \mathrm{PCO}_{2}=4,7 \pm 0,18 \mathrm{KP}, \mathrm{pH}=$ $7,39 \pm 0,03, \mathrm{Hb}=11,6 \pm 0,64 \mathrm{~g} / 1$.

L'électrocardiogramme ne s'est pas modifié, notamment sans variation de la fréquence, ni de l'espace PR, ni de la repolarisation.

Les résultats hémodynamiques sont résumès dans le tableau I et la figure 1 qui montrent, par rapport aux valeurs de base: A la cinquième minute après Bolus et sous perfusion de Diltiazem, l'absence de modification de Qc, VES, PTDVG. Par contre, on relève une baisse significative de PAOM ( -30 pour cent), PAOS ( -25 pour cent), PAOD ( -36 pour cent), dP/dt ( -30 pour cent), RAS ( -28 pour cent), WSVG ( -28 pour cent). A la dixième minute: les variables encore significativement diminuées sont la PAOM ( -12 pour cent), 

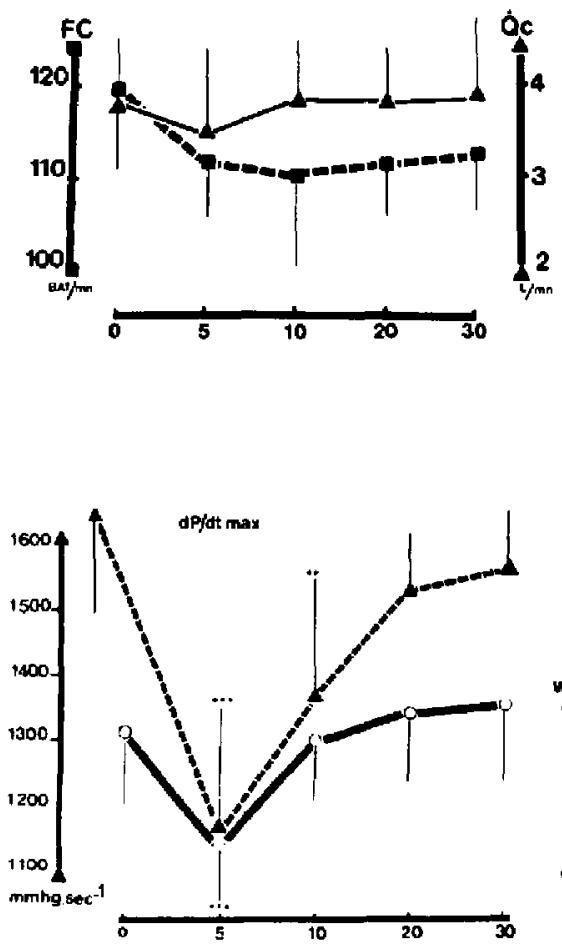

Wh.ve

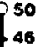

42

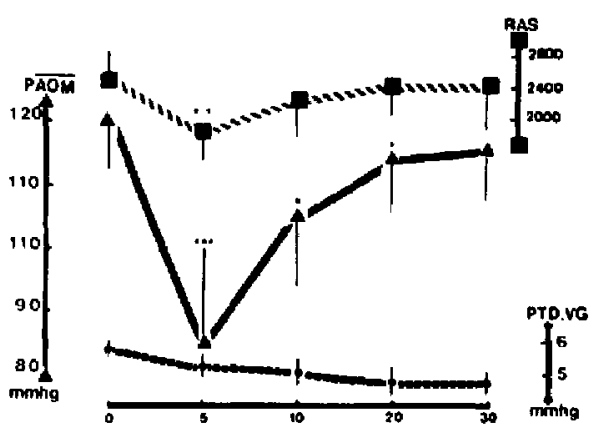

FIGURE I Variations hêmodynamiques au cours d'une anesthesie al Thiopental après bolus $\left(0,15 \mathrm{mg} \cdot \mathrm{kg}^{-1}\right)$ et sous perfusion continue $\left(0,07 \mathrm{mg} \cdot \mathrm{kg}^{-1} \cdot h\right)$ de Dilciazem. Mesures faites a $5,10,20$ et 30 minutes. (Significativité: $*$ : $p<0,05$; ${ }^{* *}: \mathrm{p}<0,01 ;{ }^{* * *}: \mathrm{p}<0,001$ ) la PAOS ( -11 pour cent), la PAOD ( -14 pour cent), la dP/dt ( -17 pour cent). Le travail ventriculaire et les résistances artérielles systémiques sont

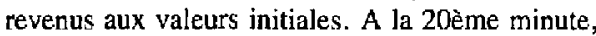
seules restent abaissées la PAOM ( -7 pour cent), la PAOS ( -6 pour cent), la PAOD ( -9 pour cent). A la 30 ème minute, l'état hémodynamique est complètement revenu à son niveau initial.

\section{Commentaires}

Ainsi, chez le porc domestique uniquement anesthésié par le Thiopental, le Diltiazem provoque pendant une courte période, à la fois une diminution de la post charge et un effet inotrope négatif.

La baisse des pressions aortiques et des résistances artérielles systèmiques, témoignent des propriétés vasodilatatrices, bien connues des agents bloqueurs calciques. ${ }^{5}$

La frequence cardiaque et la pression de remplissage du ventricule gauche êtant stables, la diminution de $\mathrm{dP} / \mathrm{dt}$ max et du travall ventricuiaire gauche témoignent d'une altération de la contractilité à la cinquième et dixième minute. ${ }^{6}$ Cependant, 1 'intensité de la variation de la pression aortique diastolique, fait perdre ici, de son acuité de la dP/dt max, comme indice de contractilité. ${ }^{7-8}$

Cet effet inotrope négatif du Diltiazem, dose dépendant, constament noté in vitro ${ }^{9-10}$ est rarement retrouvé in vivo sur les sujets conscients ou anesthésiés. Pour Flaim et Zelis, ${ }^{11}$ chez le rat conscient, le Diltiazem diminue la post charge, sans altération de la contractilité ni variation de la fréquence cardiaque, alors que le débit cardiaque tend à augmenter. Pour Millard ${ }^{12}$ chez le porc prémédiqué par la Kétamine, et anesthésié par le Thiopental et l'Halothane, l'effet inotrope négatif du Diltiazem n'est pas évident. Chez le chien anesthésié par le Pentobarbital, Bourassa et al.$^{13}$ ne. montre pas de dépression de la contractilité, après un bolus de $200 \mu \mathrm{g} \cdot \mathrm{kg}^{-1}$ de Diltiazem; la $\mathrm{dP} / \mathrm{dt}$ max ne change pas malgré une variation de post charge de même intensité que celle que nous rapportons. Cependant, cet auteur ne fait ses mesures qu'a la dixième minute, ignorant peut-être l'effet depresseur précoce que nous notons dès la cinquième minute, et dont l'intensité diminue déjà à la dixième minute, sous perfusion à débit constant.

Une interaction entre le Thiopental et le Diltiazem est donc possible. Le Thiopental et le Diltiazem sont l'un et l'autre, lié à plus de 80 pour 
cent aux protéines plasmatiques. ${ }^{14-16}$ La posologie élevée de Thiopental, nécessaire pour entretenir l'anesthésie serait responsable d'une concentration plasmatique excessive de Diltiazem lors du bolus, qui provoquerait de plus un déplacement du Thiopental vers sa fraction libre.

A dose suffisante pour l'induction anesthésique, le Thiopental provoque une dépression cardiovasculaire minime. ${ }^{17,18} \mathrm{Si}$ par voie intraveineuse directe, de hautes doses dépriment notablement les fonctions pompe et muscle, ${ }^{19-20}$ leurs effets semblent très atténués par la perfusion à débit constant. ${ }^{21}$

Sachant que les effets cardiovasculaires des barbituriques sont en partie lies à des altérations de mouvements cellulaires du calcium, une interférence à ce niveau est aussi possible. ${ }^{3,22,23}$ Lain et al. ${ }^{24}$ montre que le Thiopental réduit de 25 pour cent le captage du calcium par le réticulum sarcoplasmique. Pour Nayler et Szeto, ${ }^{3}$ il diminute le calcium endoplasmique mobilisable et accroit son stockage au niveau des microsomes. Pour Long. necker et Harris, ${ }^{25}$ l'entrée calcique ATP-dépendante est réduite et pour Altura et Altura ${ }^{26,27}$ les barbituriques inhibent les liaisons calciques membranaires et intracellulaires $\left(\mathrm{La}^{3+}\right.$ tésistantes) de 40 à 60 pour cent. Cependant à notre connaissance, aucun effet de type bloqueur calcique sur les canaux entrants lents, dépendant du potentiel d'action du myocarde, n'a jamais été mis en évidence pour le Thiopental, à la différence de ce que montre Lynch et al. ${ }^{28}$ avec l'Halothane.

Malgré la baisse des pressions aortiques et la dépression de la contractilité, provoquées par l'injection de Diltiazem pendant une anesthésie au Thiopental, la fréquence et les débits cardiaques ne varient pas. Des phénomènes "adaptatifs" sont donc mis en jeu. Sachant l'effet chronotrope négatif du Diltiazem, ${ }^{29-30}$ la stabilité de la fréquence cardiaque pour Bourassa $e t a l^{13}$ et l'absence d'effet inotrope négatif in vivo, ${ }^{11,12,31}$ résulteraient d'une stimulation sympathique reflexe, secondaire à l'hypotension engendrée par le Diltiazem. Cette stimulation sympathique du baroreflexe serait en grande partie abolie par les béta bloquants. ${ }^{32,33} \mathrm{Ce}$ reflexe fonctionne aussi lors des associations VérapamilEnflurane, ${ }^{34}$ Nifédipine-Halothane (un pour cent) pour disparaitre avec deux pour cent d'halothane. ${ }^{35}$

Avec l'Halothane et le Vérapamil, Norfleet et at. ${ }^{36}$ constate, l'absence de toute stimulation sympathique, et sa présence si on utilise le $\mathrm{N}_{2} \mathrm{O}$. Dans notre étude, la baisse prononcée de la $\mathrm{dP} / \mathrm{dt} \max$ nous ferait plûtot penser que la stimulation adrénergique n'est pas prédominante.

Ceci peut s'expliquer par l'effet conjoint de la dépression du baroreflexe par le Thiopental et des altérations du système sympathique par le Diltiazem. ${ }^{37,38}$

Les barbituriques, notamment à hautes doses, bloquent la transmission synaptique ganglionnaire $e^{39}$ en augmentant l'inhibition post symaptique ${ }^{40}$ et en deprimant les potentiels excitateurs post synaptiques. ${ }^{41}$ Ils inhibent la sécrétion de norépinephrine induite par l'entrée calcique, dépendant du potentiel d'action ${ }^{42}$ ainsi que la dépolarisation des membranes synaptosomiales dependant, du flux calcique entrant notament au niveau du tronc cérébral. ${ }^{43}$

Pour sa part, la Diltiazem inhibe les flux calciques provoqués par la stimulation alpha adrénergique, ${ }^{44}$ et au niveau préganglionnaire déprime l'activité sympathique excessive, sans affecter la transmission ganglionnaire de basc. ${ }^{38}$

La stabilité de la fréquence cardiaque serait alors la résultante de l'action chronotrope négatif, et des effets vagolytiques directe et indirect, que provoquent des agents du type Vérapamil et Diltiazem. ${ }^{30.45}$

En regard de l'altération de la contractilité, la stabilité du débit cardiaque et du volume d'éjection systolique, peut être alors attribuée à l'effet bénéfique de la diminution de la post charge ${ }^{46}$

Cette balance entre l'effet inotrope négatif et la vasodilation est retenue par Verdouw et al. ${ }^{47}$ lors de l'emploi de la Nifédipine par voie intra veineuse, et par Flaim et Zelis ${ }^{11}$ avec le Diltiazem. Ce mécanisme semble jouer un rôle important au cours de l'anesthésie, lorsque les bloqueurs calciques sont utilisés. Ainsi, au cours d'une neuroleptanalgésie, l'emploi du Vérapamil ne modifie pas le débir cardiaque malgré la baisse du travail cardiaque, ${ }^{48} \mathrm{et}$ 1'augmente dans le travail de Zimpfer et al. ${ }^{49}$ Avec deux pour cent d'Halothane, le Vérapamil provoque une chute de pression et des résistances artérielles sans variation du débit cardiaque ${ }^{35}$ Enfin, pour Hantler et al.,$^{50}$ le Vérapamil abaisse les résistances artérielles systémiques, améliore la performance cardiaque, préalablement déprimée par le Pentobarbital et l'Halothane à un pour cent. 


\section{Conclusion}

L'utilisation concomittante du Diltiazem et du Thiopental qui altère aussi les mouvements cellulaires du calcium, est susceptible de provoquer, sur le cour sain, un effet inotrope négatif transitoire. Ia diminution de post charge permet cependant une certaine stabilité de la performance cardiovasculaire. En attendant d'autres études, une certaine prudence doit donc être recommandée en fonction d'une Eventuelle pathologie cardiovasculaire, et des posologies d'utilisation de chacune des deux drogues.

\section{Bibliographie}

1 Fieckerstein A. Specific inhibitors and promoters of calcium action in the excitation contraction coupling of heart muscle and their role in the prevention or production of myocardial lesions. In: Calcium and the heart. Harris P. and Opie L. Eds. Academic Press Publ London, New York 1971: 135-88.

2 Triggle DJ. Calcium antagonists: basic chemical and pharmacological aspects. In: New perspectives on calcium antagonists. Weiss G.B. Ed. American Physiological Society, Bcthesda, Maryland 1981, $1-18$.

3 Nayler WG, Szeto J. Effect of sodium pentobarbjtal on calcium in mammalian heart muscle. Am J Physiol 1972; 222: 339-44.

4 Altura $B A$, Altura BT. General anesthetics and magnesium ions as calcium antagonists on vascular smooth muscie. In: New perspectives on calcium antagonists. Weiss G.B. Ed. American Physiological Society, Bethesda, 1981; 131-45.

5 Nagao T. Studies on a new 1,5 Benzathiazepine derivative (CRD 401) U: vasodilatation actions. Jap J Pharmacol 1972; 22: 1-10.

6 Ross $J R$, Petersan $K L$. On the assessment of cardiac inotropic state (Editorial), Circulation 1973; 47: 435-8.

7 Anderson PW, Manring A, Server CA et al. The force interval relationship of the left ventricle. Circulation 1979; 60: 334-48.

8 Broughton A, Korner PI. Estimation of maximum left ventricular inotropic response from changes in isovolumic indices of contractility in the dog. Cardioyascular Res 1981; 15: 383-9.

9 Nakajina $H$, Nosaka $K$, Hoshiyama $M$. Effects of Diltiazem on the positive inotropic and vasoconstrictor response to ouabain in vitro. Jpn J Pharmacol 1977; 27: 910-13.
10 Nabata $H$. Effects to calcium antagonistic coronary vasodilatators on myocardial contractility and $\mathrm{mcm}-$ brane potentials. Jpn J Pharanacol 1977; 27: 239-49.

11 Flaim $S F$, Zelis $R$. Effects of Diltiazem on total cardiac output distribution in conscious rat. J Pharmacol Exp Ther 1982; 222: 359-66.

12 Millard $R W$. Changes in cardiac mechanics and coronary blood flow of regionally ischemic porcine myocardium induced by Diltiazem. Chest 1980; 78 : 193-9.

13 Bourassa $M G$, Cote $P$, Theroux $P$. Hemodynamics and coronary flow following diltiazem administration in anesthetized dogs and in humans. Chest 1980; 78 (suppl): 224-80.

14 Morgan DJ, Graeme LB, Paull JD, Wolf $L J$. Pharmacokinetics and plasma binding of thiopental I: studies in surgical patient. Anesthesiology 1981; 54: $468-73$

15 Burch $P G$, Stanski DR. The role of metabolism and protein binding in thiopental anesthesia. Anesthesiology 1983; 58: 146-52.

16 Thuilliez $C$, Guidicelli JF. Pharmacologie cardiovasculaire des antagonistes du calcium. Thérapie 1981:36: 107-21.

17 Chamberlain JH, Seed RG, Chung DC. Effect of thiopentone on myocardial function. $\mathrm{Br} \mathrm{J}$ Anaesth $1977 ; 49: 865-70$.

18 Becker KE, Tonnesen AS. Cardiovascular effects of plasma levels of thiopental necessary for anesthesia. Anesthesiology 1978; 49: 197-200.

19 Sonntag $H$, Hellberg $K$, Chenk $H D$. Effects of thiopental on coronary blood flow and myocardia! metabolism in man. Acta Anaesthesiol Scand 1975; 19: $69-78$.

20 Laks $M M_{+}$Greenless L, Garner D. Comparison of ejection fraction and segmental fiber shortening velocity in the anesthetized and conscious canine. Heart J 1979; 20: 359-65.

21 Todd MM, Drummond JC, Ostrup R, Stanski DR. Hemodynamic effects of high dose thiopental anesthesia in human. Anesthesiology 1982; 57: A39.

22 Iwatsuki $N$, Yasuda I, Iwatsuki $K$. Effect of thiamylal on the sensitivity of glycerinated cardiac fibers to calcium. Br J Anaesth 1978; 50: 881-4.

23 Price HL, Ohnishi $T$. Effects of anesthetics on the heart. Fed Proc 1980; 39: 1575-9.

24 Lain RF, Hess ML, Gertz EW, Briggs FN. Calcium uptake activity of canine myocardial sarcoplasmic reticulum in the presence of anesthetic agents. Circ Res $1968 ; 23 ; 597-604$ 
25 Longnecker DE, Harris PD. Microcirculatory actions of general anesthetics. Fed Proc 1980; 39: 1580-3.

26 Altura BT, Altura BM. Pentobarbital and contraction of vascular smooth muscle. Am J Physiol 1975; 229: 1635-40.

27 Altura BM, Altura BT. Vascular smooth muscle and general anesthetics. Fed Proc 1980; 39 1584-96.

28 Lynch C III, Vogel S, Sperelakis N. Halothane depression of myocardial slow action potentials. Anesthesiology 1981; 55: 360-8.

29 Imai $Y$, Himori N. Taira N. Cardiohemodynamic effects of S.K.F. 24.260, D 600, Diltiazem, dilazep and trimetazidine in the dog. Jap Heart J 1977; 18 : 120-31.

30 Lievre M, Descoties J, Bazier JL, Timouchan $Q$, Faucon $G$. Effects of Diltiazen therapeutic plasma levels on cardiac conduction and refractoriness. Arch Int Pharmacodyn 1981; 252: 272-83.

31 Zerr C, Apoil E, Poulain D, et al. Diltiazem et anesthésie. Ann Cardiol Angéiol 1981; 30: 133-9.

32 Angus JA, Richmond DR, Dhumma-Lipakorn $P$, Cohbin LB, Goodman AH. Cardiovascular action of Verapamil in the dog with particular reference to myocardial contractility and atrioventricular conduetion. Cardiovase Res 1976; 16: 623-32.

33 Lydin $H$, Lohmoller G, Lohmoller R, Schmitz $H$, Walter I. Comparative hemodynamic studies with adalat and other antianginal drugs. 3rd Internat Adalat Symposium 1976;98-106.

34 Holland DE, Foex $P$, Francis CM, Cutfield GR. Haemodynamics of Verapamil during enflurane anaesthesia. Br J Anaesth 1982; 54: 787-8 P.

35 Tosone S, Reves JG, Kissin I, Smith LR, Fournier S, Harris $D D$. Hemodynamic interaction of Nifedipine and Halothane in dogs. Anesthesiology 1982; 57: A4 (Abst.).

36. Norfleet EA, Heath KR, Kopp VJ, Spague DH, Corke BC. Verapamil: Different cardiovascular responses during $\mathrm{N}_{2} \mathrm{O}$ analgesia and halothane anesthesia. Anesthesiology 1982; 57: A75 (Abst.)

37 Cox RH, Bagshaw RD. Influence of anesthesia on the response to carotid hypotension in dogs. Am J Physiol 1979; 237(4): H424-32.

$38 \mathrm{Ito} H$, Nishi $K$. Frequency-dependent depression of ganglionic transmission by propanolol and Diltiazem in the superior cervical ganglion of the guinea pig. Br J Pharmac 1982; 77: 359-62.

39 Barker JC, Gainer H. Pentobarbital: selective depression of excitatory post synaptic potentials. Sciences (Wash. DC) 1973; 82: 720-2.

40 Ransom BR, Barker $/ L$. Pentobarbital selectively entrances GABA mediated post synaptic inhibition in tissus cultured mouse spinal neurons. Brain Res 1976; 114: 530-5.

41 Nicoll RA, Iwamoto ET. Action of pentobarbital on synaptic ganglion cells. J Neurophysiol 1978; 41: $977-85$.

42 Haycock JW, Levy WB, Cotman CW. Pentobarbital depression of stimulus secretion coupling in brainselective inhibition of depolarization induced calcium dependent release. Biochem Pharmacol 1977; 26: $159-61$.

43 Elrod SV, Leslie SW. Acute and chronic effects of barbiturates on depolarization induced calcium influx into synaptosomes from rat brain regions. J Pharmacol Exp Ther 1980; 212: 131-6.

44 Morulsky HJ, Snavely MD, Hugues RJ, Insel PA. Interaction of verapamil and other calcium channel blockers with $\alpha 1$ and $\alpha 2$ adrenergic receptors. Circ Res 1983; 52: 226-31.

45 Karliner JS, Motulsky HJ, Dunlap J, Brown JH, Insel PA. Yerapamil competitively innibits alpha 1 adrenergic and muscarinic but not beta adrenoreceptors in rat myocardium. J Cardiovasc Pharmacol 1982; 4: 515-20.

46 Majid PA, Shama B, Tayior SH. Phentolamine for vasodilator treatment of severe heart failure. Lancet 1971; 2: 719-23.

47 Verdouw PD. Ten Cate FJ. Hugenhotz PG. Effect of Nifedipine on segmental myocardial function in the anesthetized pig. European J Pharmacol. $1980 ; 63 ; 209-12$.

48 Kates RA, Kaplan JA, McKeown PP, Freniere $S$. The cardiovascular effects of Verapamil administration during coronary artery by pass graft surgery. Anesthesiology 1982; 57: Al (Abst.).

49 Zimpfer M, Fitzal S. Tonczar L. Verapamil as a hypotensive agent during neuroleptanaesthesia. Br J Anaesth 1981; 53: 885-9.

50 Hantler $C B$, Clifford $B D$, Kroll DA, Knight $P R$. Verapamil does have prolonged interactions with Halothane. Anesthesiology 1982; 57 : A2 (Abst.).

\section{Abstract}

The clinical applications of calcium channel blockers are rapidly growing; as barbiturates interfere with intracellular calcium movements, the possiblity of drug 
inseraction as this level must be considered. We have studied the haemodynamic interaction of Thiopentone and Diltiazem in pigs. A londing dose of Diltiazem $\left(0.15 \mathrm{mg} \cdot \mathrm{kg}^{-1}\right)$ was injected followed by a continuous administration of $0.07 \mathrm{mg} \cdot \mathrm{kg}^{-1}$.hour. From the fifth to the tenth minute, the reduced afterloud was associated with a moderate depression of the contractility of the left ventricle. These values returned to the initial level within 30 minutes. Heart rate and cardiac output were not modified. The stability of these two parameters is thought to result from the complex interaction of both drugs on the sympathetic nervous system and the venticular function. 